EPJ Web of Conferences 114, 02147 (2016)

DOI: 10.1051/epjconf/201611402147

(C) Owned by the authors, published by EDP Sciences, 2016

\title{
An experimental study of the glottal jet
}

\author{
Darina Jasikova ${ }^{1, a}$, Petr Sidlof ${ }^{1}$, Michal Kotek $^{1}$ and Vaclav Kopecky ${ }^{1}$ \\ ${ }^{1}$ Institute of new technologies and applied informatics, Faculty of mechatronics, informatics and interdisciplinary studies, Technical university \\ of Liberec, Studentska 1402/2, 46117 Liberec, Czech Republic
}

\begin{abstract}
The paper presents results of the flow field analysis in a static scaled model of human larynx. Here we are focused on the effect of the nozzle gap parameter and the incoming flow velocity. The study is performed in the aerodynamic channel using the PIV technique.
\end{abstract}

\section{Introduction}

Human voice is created by expiring air from the lungs through a narrow constriction called glottis. This constriction is formed by the vocal folds, located in the larynx. The vocal folds are two symmetric soft tissue structures fixed between the thyroid cartilage and arytenoid cartilages. Under certain conditions (subglottal pressure, glottal width, and longitudinal tissue tension) the vocal folds can start to oscillate, thus modulating the flow field. The acoustic signal is created by complex aeroacoustic mechanisms in glottis and supraglottal areas, filtered by the vocal tract, radiated from the mouth, and perceived as voice.

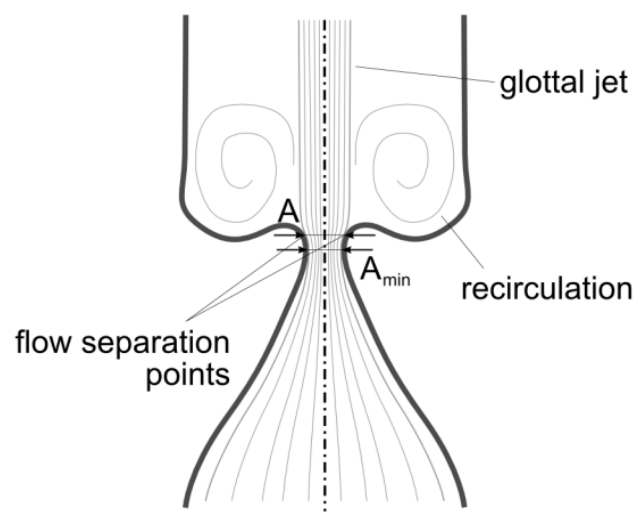

Figure 1. Idealized symmetric airflow in the glottis. Physiological orientation - flow in the inferior-to-superior direction.

From the fluid-mechanical point of view, the human larynx can be seen as a nearly planar nozzle with timevarying clearance. In the convergent part the airflow accelerates. Near the narrowest cross-section, airflow separates due to adverse pressure gradient and forms a jet (see Fig.1). The shear layer of the glottal jet generates significant vorticity in the flow field, with periodic vortex shedding creating large-scale coherent turbulent structures in the supraglottal field. Due to the interaction of the glottal jet with the vortices, the glottal jet rarely remains axisymmetric and rather teds to deflect to either of the supraglottal walls.

The concept of fluid-structure-acoustic interaction between the airflow, elastic vocal folds, and sub- and supraglottal acoustic spaces relies on knowledge of aerodynamics in the larynx. However, due to periodic closure of the glottal channel during vocal fold vibration and inherent unsteadiness of the airflow, the aerodynamic effects in the larynx are very complex. In spite of the progress in fundamental research of human voice production during recent years, some features of the glottal flow are not yet fully understood.

First glottal flow visualizations and PIV measurements were published by Triep et al. [1], who used a three times scaled hydrodynamic setup, and Erath and Plesniak [2,3] on a static 7.5 times life-size vocal fold model. Kucinschi et al. [4] confronted his Fluent computations with pressure and flow rate measurements on a mechanically driven physical model, but did not assess velocity fields. Li et al. [5] used a similar technique (with a static physical model) and tried to evaluate the flow separation points, although only qualitatively. Krane et al. [6] made measurements on an externally driven model of the human glottis in a water channel, which operated at lower frequencies.

An extensive PIV dataset on glottal flow was published by Neubauer et al. [7], who used a life-sized model of Thomson et al. [8] and quasi phase-locked PIV to measure near-field flow structures. The paper provides detailed data on jet core velocity, jet inclination angle, and also on the flow separation point, however only qualitatively. The paper of Becker et al. [9] is focused on elucidation of the mechanisms of sound production in the larynx. The authors used a synthetic life-sized selfoscillating vocal fold model. Erath and Plesniak [10] published a study on asymmetric flow features in the

\footnotetext{
${ }^{\mathrm{a}}$ Corresponding author: author@email.org
} 
glottis. Using an externally driven 7.5 times life-sized model precisely mimicking vocal fold oscillation, they quantify jet skewing in the divergent part of the glottis and deduce implications of flow asymmetries on sound production. Triep et al. [11] used an improved experimental setup equipped with time-resolved PIV to show that the supraglottal flow field is highly 3D. Their results also show that the presence of the ventricular folds decreases the pressure loss and stabilizes the jet during the divergent phase. Sidlof et al. [12] measured the position of the flow separation point during self-induced vocal fold oscillation on a scaled physical model.

Recently, Lodermeyer et al. [13] investigated the glottal jet behaviour downstream of self-oscillating synthetic vocal folds by means of phase-locked PIV technique.

In a computational study, Sidlof et al. [14] have shown that the main aeroacoustic sources are located inside glottis, and in places where the glottal jet interacts with the channel wall. The current study aims at highresolution PIV measurements of the velocity fields in domains, which are crucial for source sound generation. A scaled transparent static model of symmetric vocal folds with the "M5" shape [15] was fabricated and used for supraglottal velocity field measurements.

\section{Experimental setup}

For the purpose of this study we build the simplified model directly in the air tunnel. The air circuit was set with the narrow part of cross section $(45 \times 45) \mathrm{mm}$. In the middle of this part has been placed and fixed two segments that create the nozzle. The experimental setup of the nozzle is seen in figure 2.
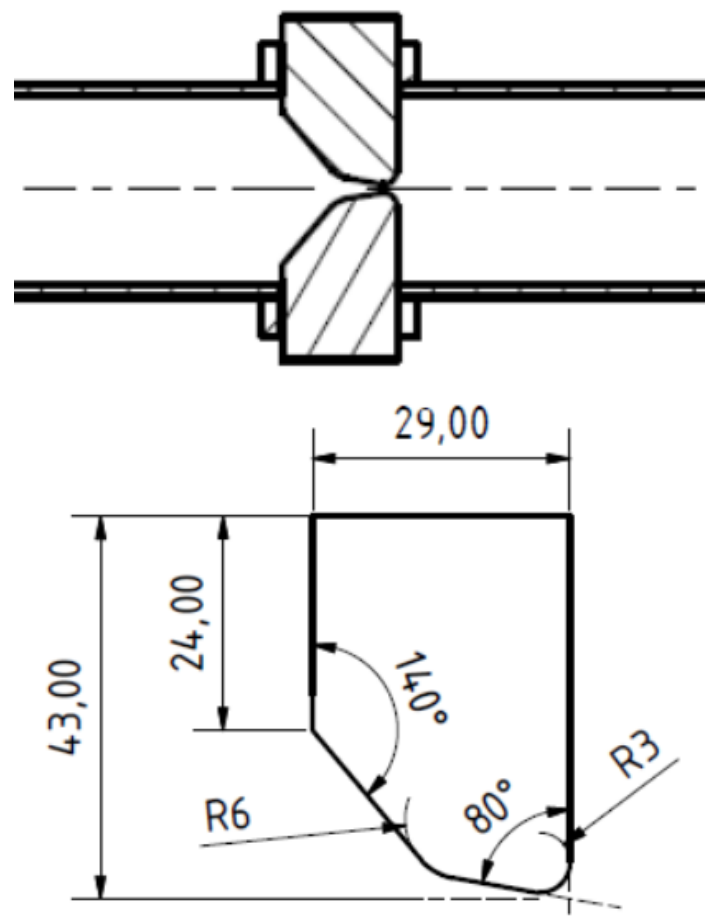

Figure 2. Experimental setup of the nozzle and the segments parameters.
Both segments were manufactured from the polycarbonate plastic and polished. The model was mounted into the chamber in the air tunnel. The nozzle gap can be adjusted. Here we used three setups: $1 \mathrm{~mm}, 2$ $\mathrm{mm}$, and $3 \mathrm{~mm}$. On the inlet to the chamber was fixed hot wire anemometry probe. The constant flow on inlet to the chamber was set by regulation on the ventilation system on the air tunnel.

The glottal jet was visualized using PIV method. The laser sheet was brought to the chamber using optical arm and the camera was fixed to the traversing unit. We recorded the flow in two different positions of camera to reach the complex view of the flow. The first view enabled capture area of interest of $(140 \times 50) \mathrm{mm}$ and the second one enabled field of view $(20 \times 20) \mathrm{mm}$ to obtain macroscopic view of the flow between the nozzles.

The PIV system consisted of NewWave Gemini pulsed laser Nd: YAG energy of $120 \mathrm{~mJ}$ per pulse length of $10 \mathrm{~ns}$, which operates at a wavelength of $532 \mathrm{~nm}$. The images were captured with Neo CMOS chip 5,5 MPixel size of 6.5 microns camera. Laser and camera system is controlled from a computer and synchronized to external TimerBox.

The flow on the inlet to the chamber was set in two steps: firstly on $0,375 \mathrm{~m} / \mathrm{s}$ and the second flow was 0,75 $\mathrm{m} / \mathrm{s}$. This velocity setup was scaled according to the scaling of the glottis model.

\section{Results and discussion}

In terms of visualization and measurement using PIV method in this task we are facing with three problems: the glottal jet represents changes in dynamic range, the laser light on the wall reflects and the nozzle represents the task of measurement in the narrow fissure.

The first problem - accelerated glottal jets against to slower moving vortex structures close to steps, has been resolved by changing of the dynamic range by adjustment method. The flow was firstly captured with the time between laser pulses optimized to slower flow that supposed to reach maximum speed of $2 \mathrm{~m} / \mathrm{sec}$. This ensured a record of slow vortex structures. In the second part of the measurement the time between laser pulses was optimized on accelerated glottal jet, wherein the expected velocity of about $8 \mathrm{~m} / \mathrm{s}$. All records were separately calculated, validated and represented as vector maps and statistics. Numerical data were sent to Matlab.

The designed algorithm combines suitable values from both vector maps. The threshold for correct values could be set by the experienced operator. More vector maps with several "time between pulses" may be combined into the resulted vector map. This procedure enlarges the low dynamical range, one of the most important limits of the PIV method.

The second problem was dealing with sufficient lightening inside the nozzle. The light was reflected to the nozzle using two inclined optical mirrors. This setup had to be precisely in geometry and the reflected light should lead directly to the source sheet, otherwise the reflection created second focusing plane that would bring the errors in the cross correlation, because of comparing different 
particles in two different measuring laser sheets. This effect was magnified in the nozzle area. The figure 3 shows the illumination of the nozzle area.

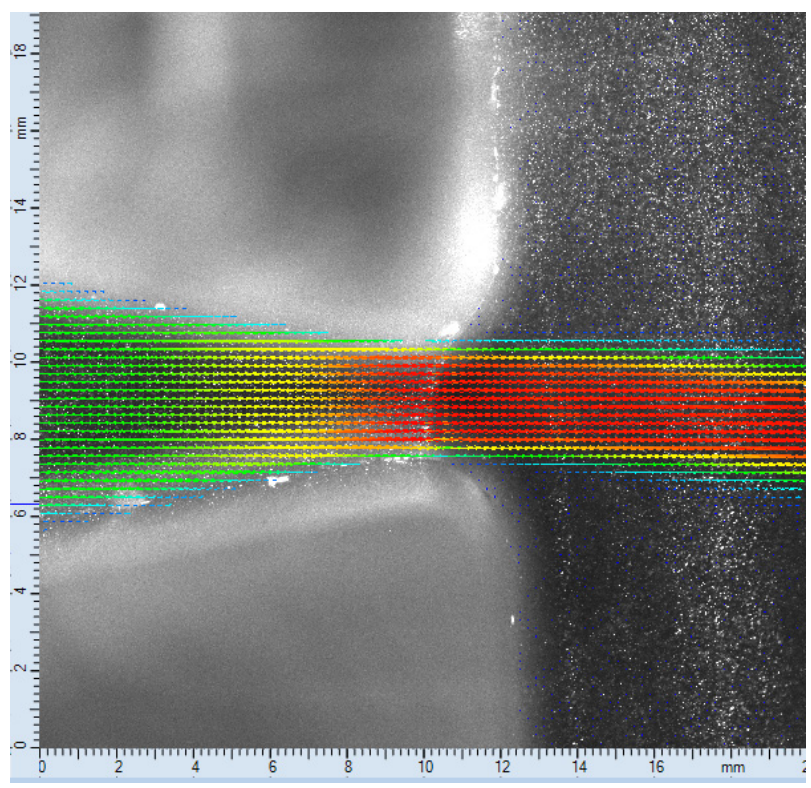

Figure 3. The magnified nozzle area under laser sheet illumination with seeding particles and calculated vector map of accelerated glottal jet.

The third problem of visualization is dealing with the illumination in the narrow structures. The segments are manufactured from the polished material that reflects and refracts the laser light, although the laser sheet is precisely focused in the sheet plane, the backward refraction bringing noise to the cross correlated vector maps. This can be solved by using image masking algorithm and image processing function that deduct all constantly refracted noise.
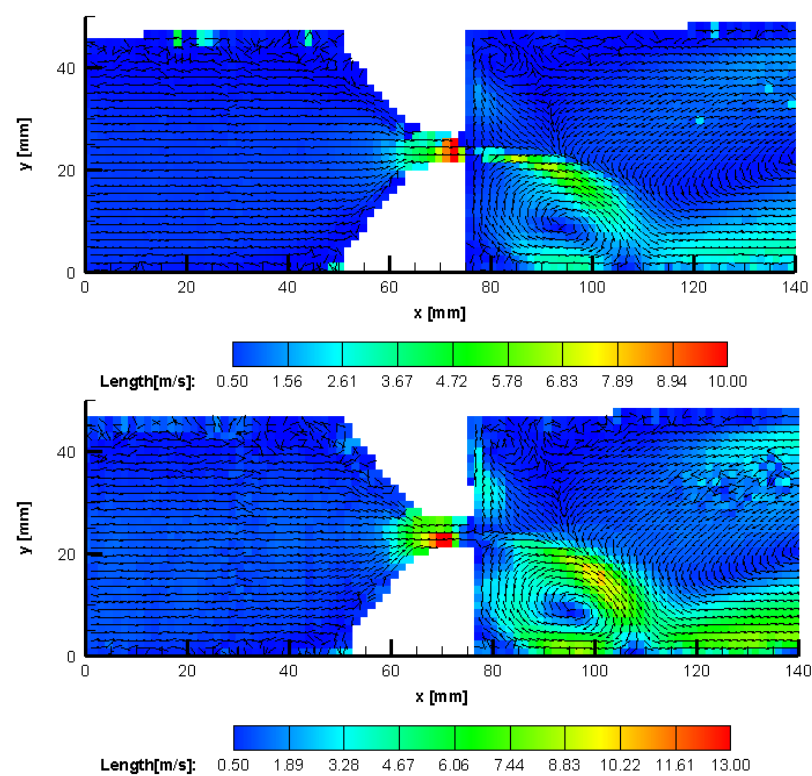

Figure 4. Vector map optimized with algorithm in Matlab position 1 with nozzle fissure $1 \mathrm{~mm}$ and incoming flow velocity $0,375 \mathrm{~m} / \mathrm{s}$ and $0,75 \mathrm{~m} / \mathrm{s}$.
Vector maps on Fig. 4 describe the flow field in the model of the glottal jet, close to the fissure with inlet velocities $0,375 \mathrm{~m} / \mathrm{s}$ and $0,75 \mathrm{~m} / \mathrm{s}$. The character of the flow stays similar in the full range of the fissure dimension. Behind the glottal nozzle the flow does not expand symmetrically, the main stream adherences to the lower wall. According the theoretical assumption the adherence should be random (upper or lower wall). Design of the model forces the stream to adhere always down. That phenomenon facilitates the statistical analysis.

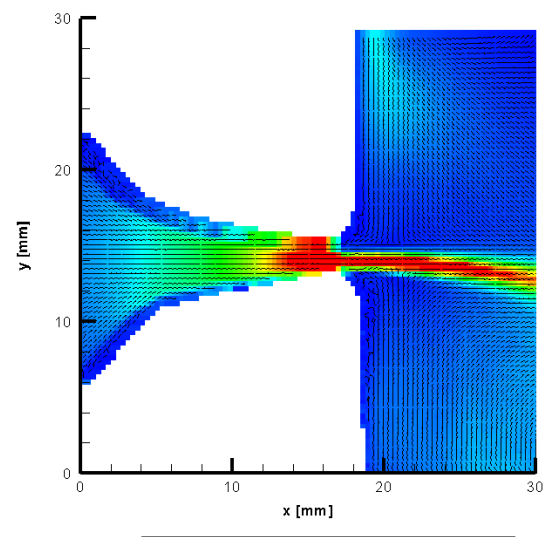

a)

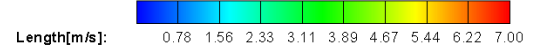

b)
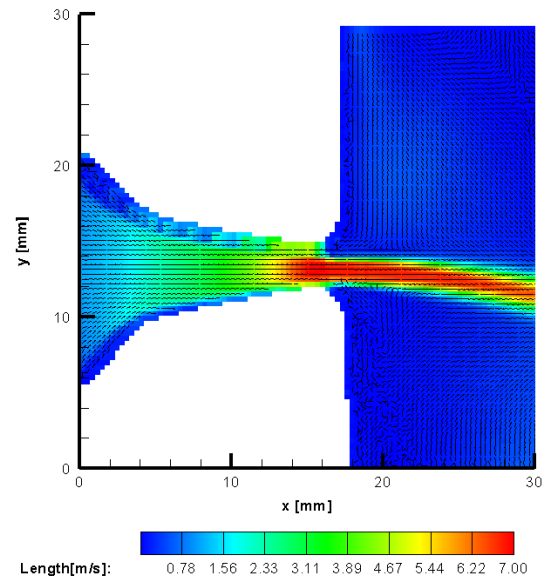

c)

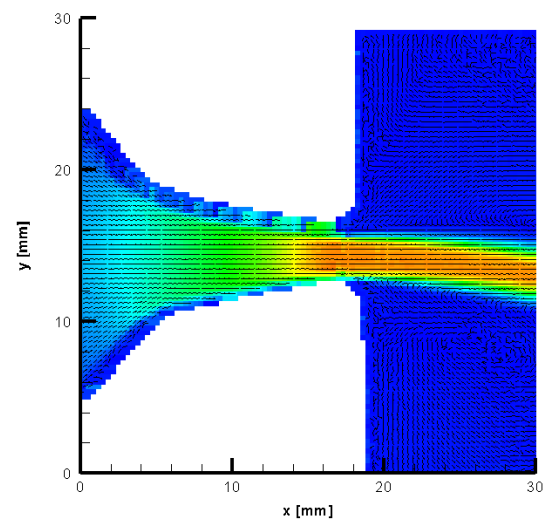

Length $[\mathrm{m} / \mathrm{s}]$

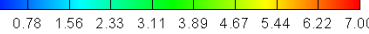

Figure 5. Vector map processed in the second position on maximal magnification. A) Calculation of the nozzle $1 \mathrm{~mm}, \mathrm{~b}$ ) for the nozzle fissure $2 \mathrm{~mm}$ and c) the nozzle $3 \mathrm{~mm}$ for the inlet flow ) $0,375 \mathrm{~m} / \mathrm{s}$. 
The stagnation point (point of the flow adherence to the wall) is found $30 \mathrm{~mm}$ (for inlet velocity $0,375 \mathrm{~m} / \mathrm{s}$ ) and $32 \mathrm{~mm}$ (for inlet velocity $0,75 \mathrm{~m} / \mathrm{s}$ ).

The series of vector maps on Fig. 5 compares the flow behind the vocal nozzle with different dimension in detail. This study proves the ability of the PIV to measure the flow in the thin vocal fissure. Using Macro Nikkon lens and HiSense Neo camera the flow profile consists of 12 vectors in $1 \mathrm{~mm}$. Analysed data are suitable to verify the numerical simulation and to set the initial conditions of the model. To get real glottal jet data, the model will be in the next step expanded with a mechanism to make the fissure oscillate.

There is seen the comparison of the velocity profiles taken in the stagnation point of the glottal jet in the figure 6 . There is seen the acceleration of the jet and maximum velocities that are increasing with the narrowing of the nozzle.
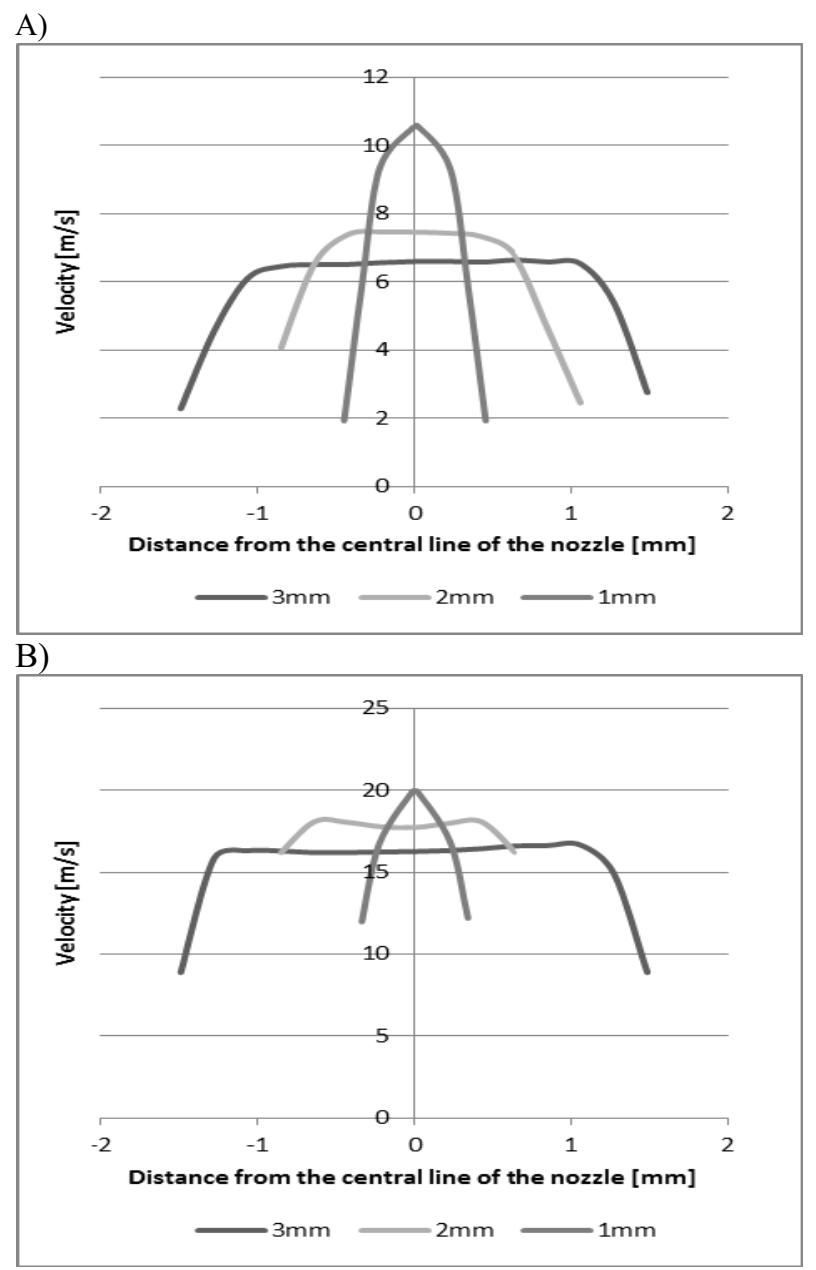

Figure 6. The comparison of the velocity profiles in the stagnation point in the nozzle for inlet flow a) $0,375 \mathrm{~m} / \mathrm{s}$ and b) $0,75 \mathrm{~m} / \mathrm{s}$

\section{Conclusions}

The concept of fluid-structure-acoustic interaction between the airflow, elastic vocal folds, and sub- and supraglottal acoustic spaces relies on knowledge of aerodynamics in the larynx. Analytical and numerical studies are run by many research teams, but the experimental data for validation and numerical model optimization are still not sufficiently provided. There are many input parameters and boundary conditions that have to be obtained. Here we started the research on the simplified scaled static model of human glottal and we applied the PIV method for accelerated glottal jet in narrow space. Following the problem of dynamic range changes we draw up the Matlab algorithm that takes in account the requirements on slow vortex structures motion estimation as well as the accelerated glottal jet. We confirmed the possibility of evaluating the flow in narrow gap. In the next step, it will be extended of the segments movement and synchronized measurement with segments oscillation in time.

\section{Acknowledgements}

The authors acknowledge the institutional support of the Faculty of Mechatronics, Informatics and Interdisciplinary Studies of the Technical University of Liberec.

\section{References}

1. M. Triep, C. Brücker,W. Schröder, Exp. in Fluids 39 (2005)

2. B. Erath, M. Plesniak, Exp. in Fluids 41 (2006)

3. B. Erath, M. Plesniak, J. of the Acoustical Society of America 120(2) (2006)

4. B. Kucinschi, R. Scherer, K. Dewitt, T. Ng, J. of the Acoustical Society of America 119 (2006)

5. S. Li, R. Scherer, M. Wan, S. Wang, H. Wu, J. of the Acoustical Society of America 119 (2006)

6. M. Krane, M. Barry, T. Wei, J. of the Acoustical Society of America 122 (2007)

7. J. Neubauer, Z. Zhang, R. Miraghaie, D. Berry, J. of the Acoustical Society of America 121 (2007)

8. S. Thomson, L. Mongeau, S. Frankel, J. of the Acoustical Society of America 118 (2005)

9. S. Becker, S. Kniesburges, S. Müller, A. Delgado, G. Link, et al., J. of the Acoustical Society of America 125, (2009)

10. B. Erath, M. Plesniak, Exp. in Fluids 49 (1), (2010)

11. M. Triep, C. Brücker, J. of the Acoustical Society of America 127(3), (2010)

12. P. Šidlof, O. Doaré, O. Cadot, A. Chaigne, Exp. in Fluids 51(1), (2011)

13. A. Lodermeyer, S. Becker, M. Döllinger, S. Kniesburges, Exp. in Fluids 56 (4), (2015)

14. P. Šidlof, S. Zörner, A. Hüppe, Biomechanics and Modeling in Mechanobiology 14 (3), (2015)

15. R. Scherer, D. Shinwari, K.D. Witt, C. Zhang, B. Kucinschi et al., J. of the Acoustical Society of America 109(4), (2001)

16. F. De Lillo, F. Cecconi, G. Lacorata, A. Vulpiani, EPL, 84 (2008)

17. L. T. De Luca, Propulsion physics (EDP Sciences, Les Ulis, 2009) 